American Journal of Applied Sciences 5 (11): 1518-1521, 2008

ISSN 1546-9239

(C) 2008 Science Publications

\title{
Speed Estimation of Adaptive Fuzzy-Controlled Piezo-Electric Motor using MLP-Neural Network
}

\author{
${ }^{1}$ Shebel ALSABBAH and ${ }^{2}$ Tariq MUGHRABI \\ ${ }^{1}$ Department of Mechatronics and ${ }^{2}$ Department of Computer Engineering, \\ Faculty of Engineering Technology (FET) \\ Al Balqa Applied University (BAU), PO Box 15008, Amman - Jordan
}

\begin{abstract}
The speed of ultrasonic motor of piezo-electric type is usually measured using mechanical sensors such as pulse encoders. However, these sensors are costly and bulky. In this paper, a numerical speed estimation approach of a piezo-electric motor (PEM) is implemented using multilayer perception neural network (MLP-NN). The proposed model evaluates rotational speed and load torque based on the amplitude and driving frequency of the terminal voltage, considering the temperature variation. The estimated speed is employed to enhance the performance of the adaptivefuzzy based speed control system. The model is validated and examined to achieve a minimized relative error in speed estimation approaches.
\end{abstract}

Keywords: Neural Network, MATLAB, USR60, hybrid model

\section{INTRODUCTION}

Since the last two decades, speed-sensorless control methods of different motors using the estimated speed instead of measured speed have been studied. Recently, the use of neural networks NNs to identify and control non-linear dynamic systems such PEM has been proposed because they can approximate a wide range of non-linear functions to any desired degree of accuracy. Moreover, they have the advantage of extremely fast parallel computation.

The networks of neurons (NN) are strongly connected assemblies of calculating units. The latter originate in a biological model of neuron, of which they retain only one extremely simplified vision.

The first model was proposed in 1943 by W.S. Mc Culloch and W. Pitts. The latter supposed that the nervous impulse was the expression or the result of a simple calculation carried out by each neuron and that it is thanks to the collective effect of a network of interconnected neurons that is born the thought ${ }^{[1,2]}$.

From different view, the piezo-electric motors (PEMs) have structural and operational advantages compared to conventional electromagnetic motors, such as compact size, lighter weight, very low speed operation, high torque, nonmagnetic operation, freedom of constructional design, very low inertia, high speed response, possibility of electromagnetic noise reduction and miniaturization. However, some important technical problems remain to be solved in the context of large-scale industrial adoption of PEM related to efficiency, performances, speed estimation and precise control. That's why; this study attempts to deal with designing of MLP-neural network- based speed estimator to be then fed back in the speed control loop of PEM where we can find there the self-tuned fuzzy PID control algorithm.

\section{STRUCTURE OF THE PROPOSED MLP-NNSE}

The physical problem which we deal with is non linear, that is why a non linear multi- layer neural network MLP-NN has been chosen. The structure used in this work for this type of application constitutes of one hidden layer with a hyperbolic tangent activation function and output layer with linear function as shown in Fig. 1.

For such problems, where the MLP-NN is proposed to estimate the rotary speed at the motor shaft, there is no general method to fix the architecture of the network (number of neurons in the hidden layer).

In this case, we are going to study certain number of neuronal architectures. For each architecture, we do different initializations of synaptic parameters to assure that the training of the NN converges towards the total minimum of the error criterion. For each structure, we calculate the mean square error MSE in the training and

Corresponding Author: Shebel Alsabbah, Department of Mechatronics, Al Balqa Applied University (BAU), P.O. Box 15008, Amman, Jordan Tel. 0096264892342 


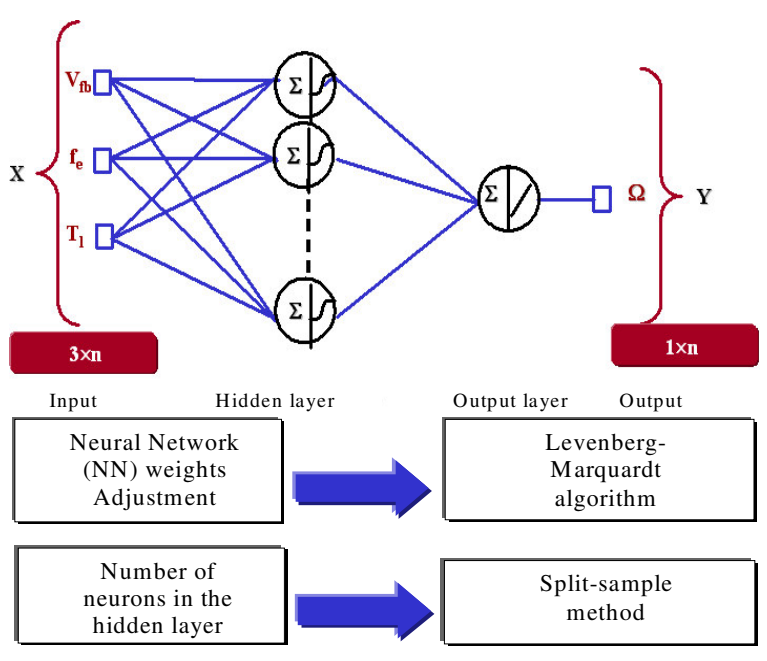

Fig. 1: Structure of the proposed MLP-NNSE

validation data bases. Then, the adequate structure that we are concerned is the structure which has the least square error in the validation base.

\section{PEM SPEED ESTIMATION USING MLP-NN}

Using the thermo-electromechanical hybrid model derived in $^{[3]}$, both the PEM rotation speed $\Omega$ and the load torque $\mathrm{T}_{1}$ could now be estimated.

The training algorithm used in this work is the conventional backpropogation algorithm ${ }^{[4]}$.

The main three algorithms (training, validation and testing) of the $\mathrm{NN}$ speed estimator are presented as in Fig.2.

\section{EVALUATION OF RESULTS}

In order to assess the performance of the proposed initialization method, some experiments have been performed. Both the drive and the feedback characteristics of Shinsei motor (USR60) ${ }^{[5]}$ had been examinated at different operating conditions (Fig.3: a-c).

In Fig. 3a, it could be noticed that the drive characteristics (the mechanical rotary $\Omega$ speed versus the control variable f) effected by the temperature variation, in other words, when we work at constant frequency, the speed increases when the working temperature increases.

While, Fig. 3b shows the feedback frequency in function of the motor speed at load changes $T_{1}$ and finally, Fig. $3 c$ shows the FB frequency $f_{f b}$ in function of actual temperature $\theta$.
Step 1: Read all input scalars of NNE and output vectors from full thermo-electromechanical model $\left(\mathrm{W}_{1}, \mathrm{~W}_{2}, \mathrm{~B}_{1}, \mathrm{~B}_{2}\right.$, mean_p, mean_T, et_p, et_T, $S_{1}$ and $\left.f_{\mathrm{FB}}, V_{\mathrm{fb}}, \theta\right)$

Step 2: Initialize the three main vectors presenting the split-sample method (training, validation and testing datas bases)

Step 3: Conditioning of $\mathrm{P}$ for the three main basis

Step 4: For the hidden layer, calculate the net input and output as shown in the internal structur of the MLP-NN in Fig. 1

Step 5: Is the difference between target and output pattern within tolerance?

Step 6: For each output unit calculate the error and update the weights.

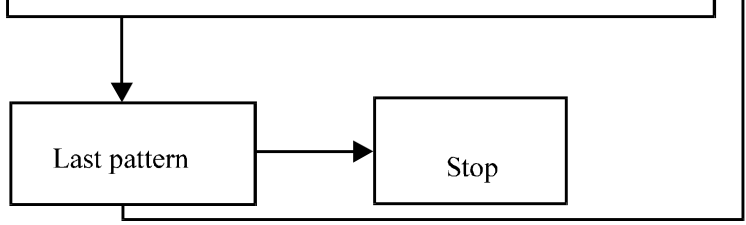

Fig. 2: Flow chart presents the $\mathrm{NN}$ three main algorithms (training, validation and testing)

Those experimental results could be then used:

- To design the inputs/output states of the MLP-NN speed estimator

- To create the data bases for the three main algorithms in the NN (i.e. Training, validation and testing)

\section{EVALUATION OF THE MLP-NN PERFORMANCE}

Figure 4 presents the evaluation of the minimal MSE in the validation data base had (green colour) been used to define the number of neurons in the hidden layer (in our case is equal 2) for the chosen structure.

\section{INTERPRETATION OF RESULTS}

Finally, the capacity of the net will be tested to find the estimated speed that corresponds the least error 


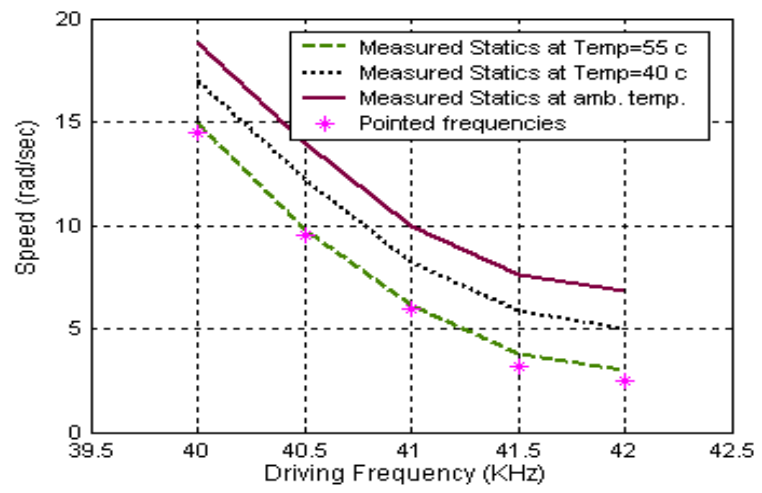

(a)

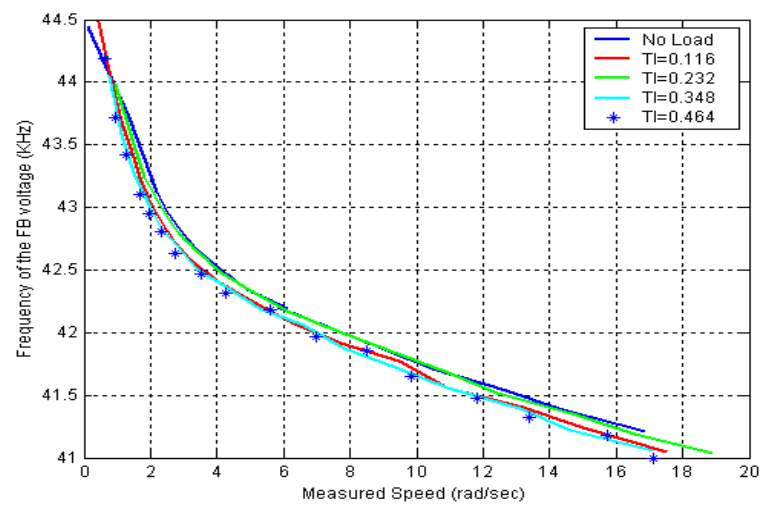

(b)

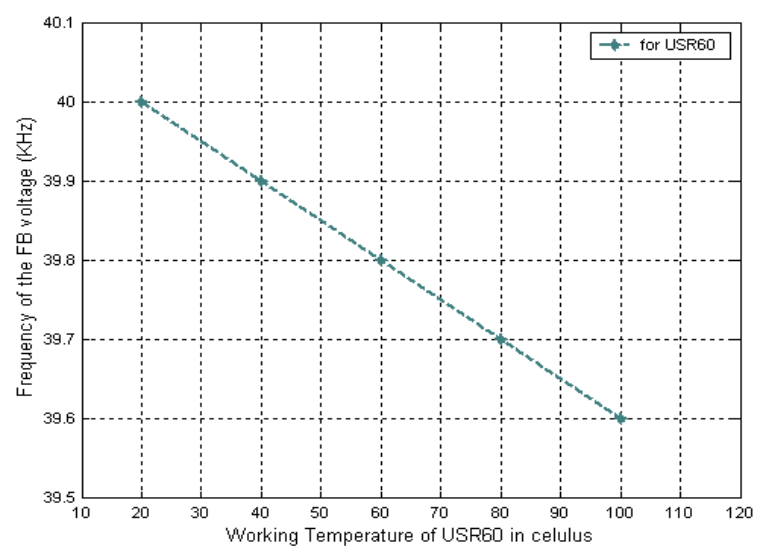

(c)

Fig. 3: Performed experimental results for USR 60

between the estimated and real speed for different values of feedback voltages $V_{f b}$ and frequencies $f_{f b}$. The utilized examples are of different values than the precedent ones (training and validation data base).

The error between the real and estimated speed values is defined for each parameter by the relative error $\operatorname{ER}(\Omega)$ calculated by the following formula 1 ,

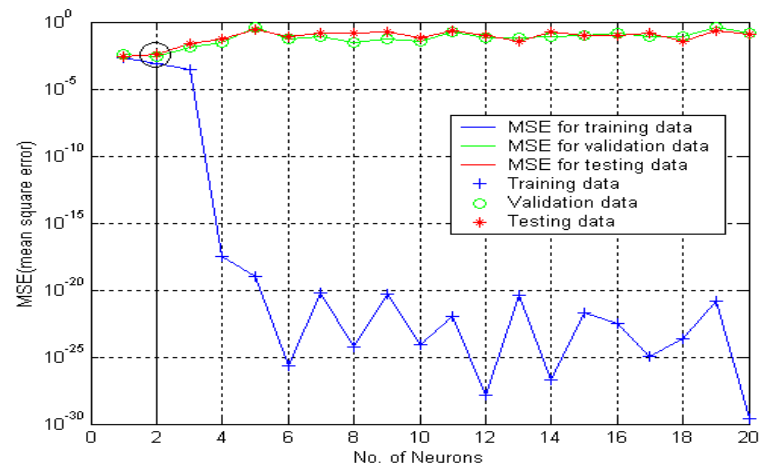

Fig. 4: MSE for both the training and validation data bases

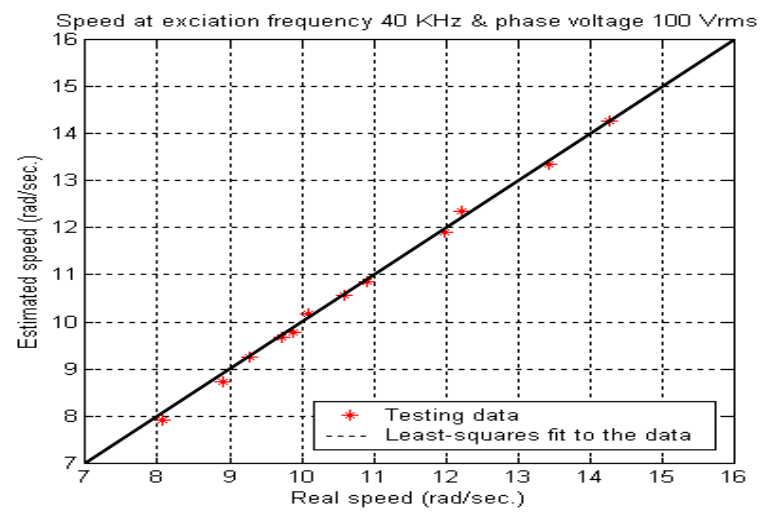

Fig. 5: Estimated parameters in function of real ones

$$
\operatorname{ER}(\Omega)=\sqrt{\frac{1}{N} \sum_{i=1}^{N}\left(\frac{\hat{\Omega}_{i}-\Omega_{i}}{\Omega_{i}}\right)^{2}}
$$

where, $\mathrm{N}$ is the number of considered points (in our case is equal 12), as will be shown below in Fig. 5. $\hat{\Omega}_{\mathrm{i}}$ is the estimated speed at each input testing parameters and $\Omega \mathrm{i}$ is the real speed.

For the proposed design, it had been found that the relative error is $0.02 \%$, which is accepted. So the designed MLP-NNSE has a sufficient values of accuracy. Those calculations are generalized and illustrated below in Fig.5,

\section{AFPID CONTROLLER WITH MLP-NNSE}

This section presents the proposed structure of the PEM controlled by self-tuning fuzzy PID system STFPID with speed estimation using MLP-NNSE, see Fig.6. 


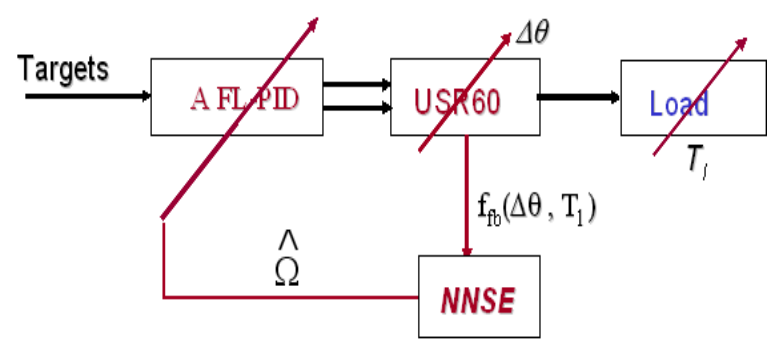

Fig. 6: Structure of the AFPID-controlled PEM using MLP-NNSE

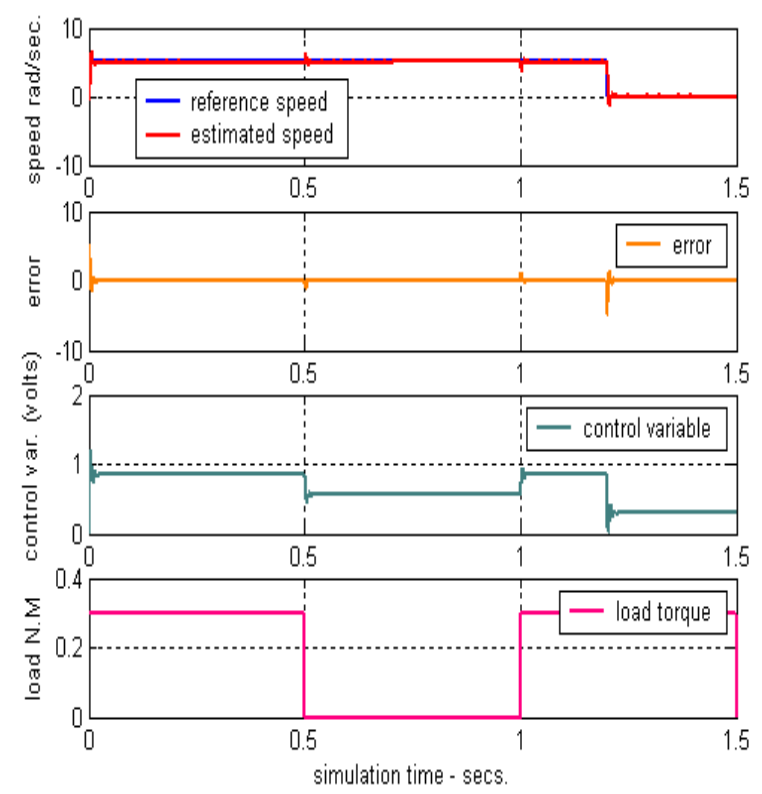

Fig. 7: Evaluation of comparative results

Using MatLab, the structure shown in Fig. 6 has been performed and the numerical results are as shown in Fig. 7. They present the performance of the AFPIDcontrolled PEM for both cases:

(a) Speed calculation using the simplified hybrid model SHM derived in ${ }^{[3]}$

(b) And speed estimation using the proposed MLP-NN

\section{CONCLUSION}

From all mentioned above in this paper, it could summarize the general conclusions in few points as follow:

- With MATLAB computational language and iteration algorithm, the rotation speed and load torque versus input parameters (input voltage amplitude and driving frequency) could be estimated. The estimated speed is then fed back in the speed control loop, taking into account the temperature variation

- The structures of the proposed MLP NN speed estimator had been designed of 2 neurons in single hidden layer of continuous and non-linear functions (such hyperbolic tangential function HTF) and of linear function LF at the output layer

- From the driving characteristics shown in Fig. 3a, it could emphasize that the effect temperature should be integrated in the designed model

- Despite the tolerance appears between the two curves for the training and validation data shown in Fig. 4, it should be emphasized that we are dealing here with highly non-linear system (PEM). On other side the relative error found is very acceptable for such system $(0.02 \%)$

Finally, from the testing data we could obtained, a stable and efficient adaptive FLC of PID type with utilization of MLP-NN speed estimator for the piezoelectric motor PEM. The proposed models had been designed and validated numerically.

\section{ACKNOWLEDGEMENTS}

I would like to acknowledge all who save to me different tools and ideal working environment to finally produce such work.

\section{REFERENCES}

1. Callan, R., 1998. The Essence of Neural Networks. The Essence of Computing Series, Prentice Hall Europe, pp: 20-32.

2. Li, W., X.G. Chang, F.M. Wahl and S.K. Tso, 1999. Hybrid Fuzzy P+ID Manipulators under Uncertainty. Elsevier Science Ltd. 4, 1 (1): 301-315.

3. Alsabbah, S.A., E. Mendes and Y. Bernard, 2003. Derivation of simplified hybrid model and speed control for travelling wave ultrasonic motors. Int. J. Applied Electromagnetics and Mechanics, IJEM2003, Ref.JAE632.

4. USR60, shinsei industry, Shinsei Motor Data Sheet.

5. Giraud, F., B. Semail and J. Audren, 2004. Analysis and phase control of a piezoelectric traveling-wave ultrasonic motor for haptic stick application. Transactions on Industry Appl., 40 (6,1 (1)): 1541- 1549. 